\title{
Electronic Structure of Cdse Nanowires Terminated With Gold Electrodes
}

\section{Hagos Woldeghebriel}

Department of Physics, College of Natural and Computational Sciences, P.O.Box 231, Mekelle University, Ethiopia (hagos93@mu.edu.et)

\begin{abstract}
Cadmium selenide nanowires in the wurtzite bulk phase, connected to gold electrodes are studied using local density approximation. The short wire is fully metalized by metal-induced gap states. For longer wires, a gap similar to that in bare cadmium selenide nanowires is observed near the center while sub-gap structure emerges near the metal-semiconductor nanocontact. This behavior is attributed to the formation of sub-gap interface states that vanish rapidly towards the center of the wire, consistent with experimental results. For the longer wires Schottky barriers develop with heights larger than the corresponding bulk value. Our study thus indicates that the length of the naonowire sandwitched between gold electrodes should be greater than $8.76 \AA$ to get characteristics of bare CdSe nanowire.
\end{abstract}

Key Words: Metal-induced gap states, Schottky barrier, Nanodumbell, Nanocontact, Gold electrodes, CdSe nanowire

\section{INTRODUCTION}

Understanding electron transport through nanoscale junctions or molecular devices connected to metallic electrodes may be the basis of future molecular electronics technology (Nitzan, 2001). One of the critical issues in this regard is to construct contact structures which can provide both useful stability and high contact transparency. Compared with the traditional semiconductor (SC) technology this new approach would change both the operating principles and the materials used to fabricate the devices.

It was first suggested in the early 1970s by Aviram and Ratner that such a system should have the ability to rectify current (Aviram and Ratner, 1974). When electric current flows through a single molecule, the conductance is mainly decided by the quantized electronic structure of the molecule. The presence of accessible charge states near the electrode's Fermi level can help electron transport through a molecule. The properties of the contacts between the molecule and the leads are also important and they strongly affect the overall conductance of a single molecule device. Control over the electronic and optical properties of semiconductors (SCs) is a major goal in science and technology. SC nanoparticles provide such an opportunity, due to the threedimensional size quantization they exhibit leading to modification of their electronic properties (e.g., the band gap) with particle size. 
Understanding the factors which determine the size and structure of SC nanoparticles is therefore, important in order to rationally control their optoelectronic behavior. Owing to their unusual electronic and structural properties, SC clusters have received considerable attention (Dabbousi et al., 1995; and Troparevsky et al., 2001). A remarkable characteristic of SCs is the evolution of their physical and chemical properties with size. Clearly, the role of the surface and surface induced properties becomes increasingly important for small systems.

Owing to their size dependent photoluminescence that is tunable across the visible spectrum, CdSe nanocrystals are attracting increasing attention for applications such as fluorescence tags for biological molecules, raw materials for tunable LEDs, optical memory, and so on (Deglmann et al., 2002; Nir et al., 2003; Xia et al., 2004; Csik et al., 2005; Yu et al., 2006; and Wu et al., 2007). Significant progress has been made in recent years in fabricating and understanding the physical properties of single phase shape-controlled SC nanostructures (Thomas et al., 2005; and Millo et al., 2001). However, the research of hybrid metal-SC nanostructured systems is still in its infancy. A fundamental and intriguing problem associated with such systems is the mechanical and electrical properties of metal-SC nanojunctions. In bulk systems, such an interface is characterized by the space charge region and the corresponding Schottky barrier. The spatial extent of this interface region is typically tense of $n m s$, comparable, and in many cases larger than many SC nanostructures prepared today. Hence, the bulk concepts for the metal-SC interface should be revisited for nanoscale contacts.

From the theoretical point of view, understanding the properties of such nanocontacts is a major step in the route towards the implementation of SC nanocrystals in nanoelectronic device architecture.

The metal-SC nanojunction was treated theoretically by Landman et al. (2000) who studied the nanocontacts formed between Si-nanowires and $\mathrm{Al}$ nanoelectrodes. These authors predicted the induction of sub-gap states near the $\mathrm{Si}-\mathrm{Al}$ interface, which rapidly decay into the $\mathrm{Si}$, and the development of relatively large Schottky barrier.

Experiments on molecular wires have included studies of conduction in molecular thin films (Zhou et al., 1997), in self-assembled monolayers (SAM's) using a scanning tunneling microscope (STM) (Stipe et al., 1997), and through a single molecule connected between the tips of a mechanically controlled break junction (Reed et al., 1997). Recently, electronic properties of metal-SC nanojunctions are investigated by scanning tunneling spectroscopy of gold-tipped 
CdSe rods (Steiner et al., 2005). A gap similar to that in bare CdSe nanorods (Katz et al., 2002) is observed near the nanodumbell (NDB) center, while sub-gap structure emerges near the metalSC nanocontact. They have also demonstrated that the NDBs provide a unique model system to study the physical properties of metal-SC nanojunctions, a fundamental problem of significant importance in the quest for reliable nanoelectronic devices. Motivated by the practical application of the metal-SC hybridization and the limited number of works in this area, I present a theoretical study of the nanocontacts formed between CdSe nanowires and Au nanoelectrodes. The systems studied in this work are of the form $\mathrm{Au}_{13}-\mathrm{Cd}_{n} \mathrm{Se}_{\mathrm{n}}-\mathrm{Au}_{13}$ nanocontacts, placed the $\mathrm{Cd}_{n} \mathrm{Se}_{\mathrm{n}}$ nanowire between two $\mathrm{Au}_{13}$ electrodes.

\section{COMPUTATIONAL DETAILS}

The calculations are based on a plane wave expansion method employing relativistic ultrasoft pseudopotentials (USPP) (Vanderbilt, 1990; and Kress and Furthmuller, 1996) and the local density approximation (LDA) for exchange and correlation term approximated by the CeperleyAlder functional (Ceperley et al., 1980) and parametrized by Vosko-Wilk-Nusair (Vosko et al., 1980). A super cell approach is used with imposition of periodic boundary conditions and the reciprocal space is sampled by the $\Gamma$ point. All calculations described in this work are performed with the Vienna $a b$ Initio Simulation Package (VASP) (Kress et al., 1996). Structural optimizations are performed using the conjugate gradient (CG) method with force convergence criterion of $0.01 \mathrm{eV} / \AA$ and energy convergence of $10^{-4} \mathrm{eV}$ for electronic self-consistency. A sufficiently large unit cell is chosen so that the minimum distance from the cluster boundary to cell boundary is about $6 \AA$.

The geometry of $\mathrm{Au}_{13}$ cluster (shown in Fig. 1 (a)) considered in the study is generated by performing molecular dynamics simulations. A similar geometry has been reported earlier (Shafai et al., 2007; and Fernandez et al., 2004) but it differs from others (Furche et al., 2002; Wang et al., 2002; and Gilb et al., 2002). The CdSe nanowires are generated by relaxation of fragments, $\mathrm{Cd}_{6} \mathrm{Se}_{6}$, Cd10Se10 and $\mathrm{Cd}_{14} \mathrm{Se}_{14}$, of the wurtzite structure of CdSe crystal. The valence electron configurations used are $\left(5 d^{10} 6 s^{1}\right)$ for $\mathrm{Au}, 4 \mathrm{~d}^{10} 5 \mathrm{~s}^{2}$ for $\mathrm{Cd}$ and $4 \mathrm{~s}^{2} 4 \mathrm{p}^{4}$ for Se atoms. A plane wave cut-off of $179.7 \mathrm{eV}$ is used for bare gold cluster and the $\mathrm{Au}_{13} \mathrm{Cd}_{\mathrm{n}} \mathrm{Se}_{\mathrm{n}} \mathrm{Au}_{13}$ lead-nanowire structures while $167.9 \mathrm{eV}$ is used for bare CdSe nanowires. 


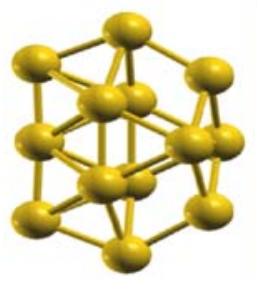

(a)

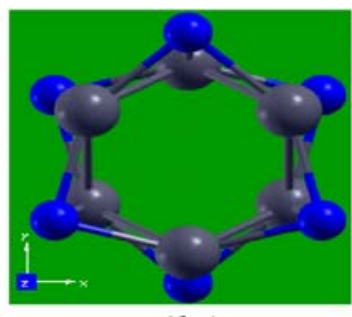

(b)

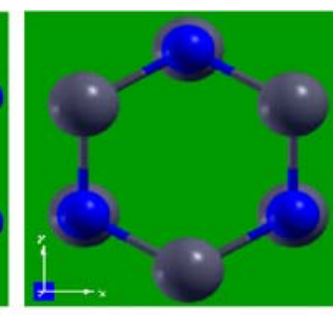

(c)

Figure 1. Geometry of $\mathrm{Au}_{13}$ (a), relaxed (b) and unrelaxed (c) of $\mathrm{Cd}_{6} \mathrm{Se}_{6}$ cluster along with a coordinate system describing the direction for the growth of the nanowire. In this study the contact of $\mathrm{Cd}_{n} \mathrm{Se}_{n}$ with the $\mathrm{Au}_{13}$ cluster is along the $\mathrm{x}$-axis (from right direction of (a)).

The contact between the $\mathrm{Au}_{13}$ lead and the $\mathrm{Cd}_{n} \mathrm{Se}_{\mathrm{n}}$ nanowire is through four atoms from both sides, lying almost in a plane perpendicular to the length of the nanowires. We refer to these lead-nanowires as $\mathrm{Au}_{13} \mathrm{Cd}_{n} \mathrm{Se}_{\mathrm{n}}$ nanodumbells.

It is a usual practice to passivate clusters to satisfy the dangling bonds at the surface (Manna et al., 2005; and Settler et al., 2002). However, CdSe nanostructures have been shown to be relatively insensitive to passivation effects (Puzder et al., 2004).

\section{RSULTS AND DISCUSSION}

\subsection{Au-CdSe Nanocontact}

In the coming sections, we will be presenting results for Au-CdSe nanodumbells of the form $\mathrm{Au}_{13} \mathrm{Cd}_{n} \mathrm{Se}_{n} \mathrm{Au}_{13}$, $(n=6,10$, and 14$)$, herein after they will be designated as $\mathrm{Au}_{13} \mathrm{Cd}_{n} \mathrm{Se}_{\mathrm{n}}$ nanodumbell. We define the binding energy $B E$ of the structures as

$B E=2 \mathrm{E}\left(\mathrm{Au}_{13}\right)+\mathrm{E}\left(\mathrm{Cd}_{\mathrm{n}} \mathrm{Se}_{\mathrm{n}}\right)-\mathrm{E}\left(\mathrm{Au}_{13} \mathrm{Cd}_{\mathrm{n}} \mathrm{Se}_{\mathrm{n}} \mathrm{Au}_{13}\right)$

\subsection{1. (a) $A u_{13} C d_{6} S_{6}$ Nanocontact}

Geometry of $\mathrm{Au}_{13} \mathrm{Cd}_{6} \mathrm{Se}_{6}$ nanodumbell is shown in Fig. 2 (a). In the $\mathrm{Au}_{13} \mathrm{Cd}_{6} \mathrm{Se}_{6}$ nanodumbbell a slight outward relaxation of the atoms is found around the junction as compared to that of the bare $\mathrm{Au}_{13}$ and $\mathrm{Cd}_{6} \mathrm{Se}_{6}$ clusters.

A similar elongation of Au-Au bond lengths for those Au atoms bonded to sulfur are found by Larsson (Larsson et al., 2002). Average Au-Cd bond length, $2.70 \AA$ A, is greater than that of Au-Se bond length, $2.62 \AA$, due to the larger radius of the $\mathrm{Cd}$ atom. Both $\mathrm{Cd}$ and Se atoms are doubly 
bonded to the Au atoms but $\mathrm{Cd}$ atoms have more near neighbors than the Se atoms.

The binding energy calculated using equation above and the HOMO-LUMO gap energy are $7.854 \mathrm{eV}$ and $0.314 \mathrm{eV}$, respectively. The difference between the Fermi energy, $\mathrm{E}_{\mathrm{F}}$, and the position $\mathrm{E}_{\mathrm{c}}$ of the bottom of the conduction states is calculated to be $0.18 \mathrm{eV}$.

Some insight into the electronic transport properties of molecular devices can be obtained by studying the local density of states (LDOS) of the nanodumbell. Although the LDOS does not fully determine the zero-bias conductivity, regions where the LDOS is depressed are expected to be barriers for electronic flow (Piccinin et al., 2003). Figs. 2 (b) display LDOS of the $\mathrm{Au}_{13} \mathrm{Cd}_{6} \mathrm{Se}_{6}$ nanodumbell. Information of the metallicity of the cluster can be inferred from LDOS plots as a function of the distance from the metal-SC junction to the center along the axis of the nanowire. (A) of Fig. 2 (b) is the LDOS of the whole $\mathrm{Au}_{13} \mathrm{Cd}_{6} \mathrm{Se}_{6}$ nanodumbell. In this figure, there is a broader deep around an energy of $-4 \mathrm{eV}$. States stemming from $\mathrm{Cd}(d)$ levels are seen suppressed here.

(I) - (III) are LDOS calculated in different regions as defined in the geometry shown in (a). There are two CdSe dimers in each of the regions (II) and (III). On top of the LDOS of $\mathrm{Au}_{13} \mathrm{Cd}_{6} \mathrm{Se}_{6} \mathrm{NDB}$ is shown the LDOS at the edge (E) and center (C) of bare $\mathrm{Cd}_{6} \mathrm{Se}_{6}$ nanowire plotted for comparison. The discrete spectra were broadened by a Gaussian of width $0.02 \mathrm{eV}$. The vertical line denotes $\mathrm{E}_{\mathrm{F}}$.

In the same figure is the LDOS of the four Au atoms, (I), at the junction, where there is a similar characteristics with that of (A). In (II) is the LDOS of CdSe atoms in contact with the Au lead. Still there is no significant difference between the forms of states in (I) and (II) LDOS plots. This is an indication that the CdSe atoms in contact with the Au atoms are metalized. And in (III) is the LDOS of CdSe atoms at the center of the nanowire. Now there is a gap widening in (III) as compared to the gap in (II), but still there are mid-gap states (MGS) created in the gap of (III). The local density of states reveals a finite DOS in the gap both in the region of the wire bonded directly to the Au electrode (II) and the one in the middle of the cluster bridge (III). We can therefore say that the $\mathrm{Cd}_{6} \mathrm{Se}_{6}$ nanowire shows metallic property as it is sandwiched between $\mathrm{Au}_{13}$ nanoelectrodes. The metalization of the CdSe nanowire bridging the Au electrodes is by the metal-induced-gap states (MIGS) (Landman et al., 2000). The length of the $\mathrm{Cd}_{6} \mathrm{Se}_{6}$ nanowire measured between the interfacial Cd/Se atoms bonded to the Au electrodes is $4.62 \AA$. 


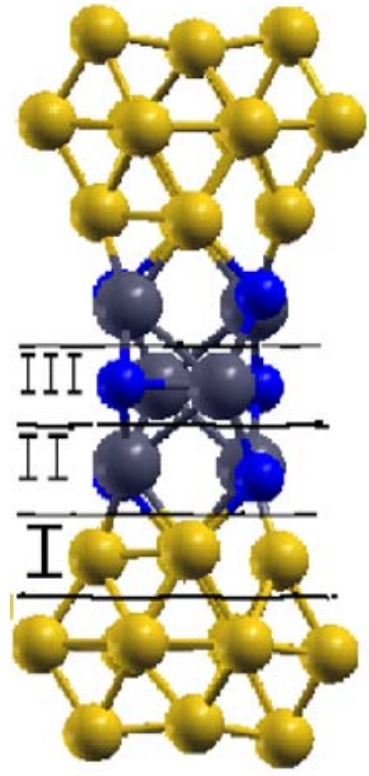

(a)
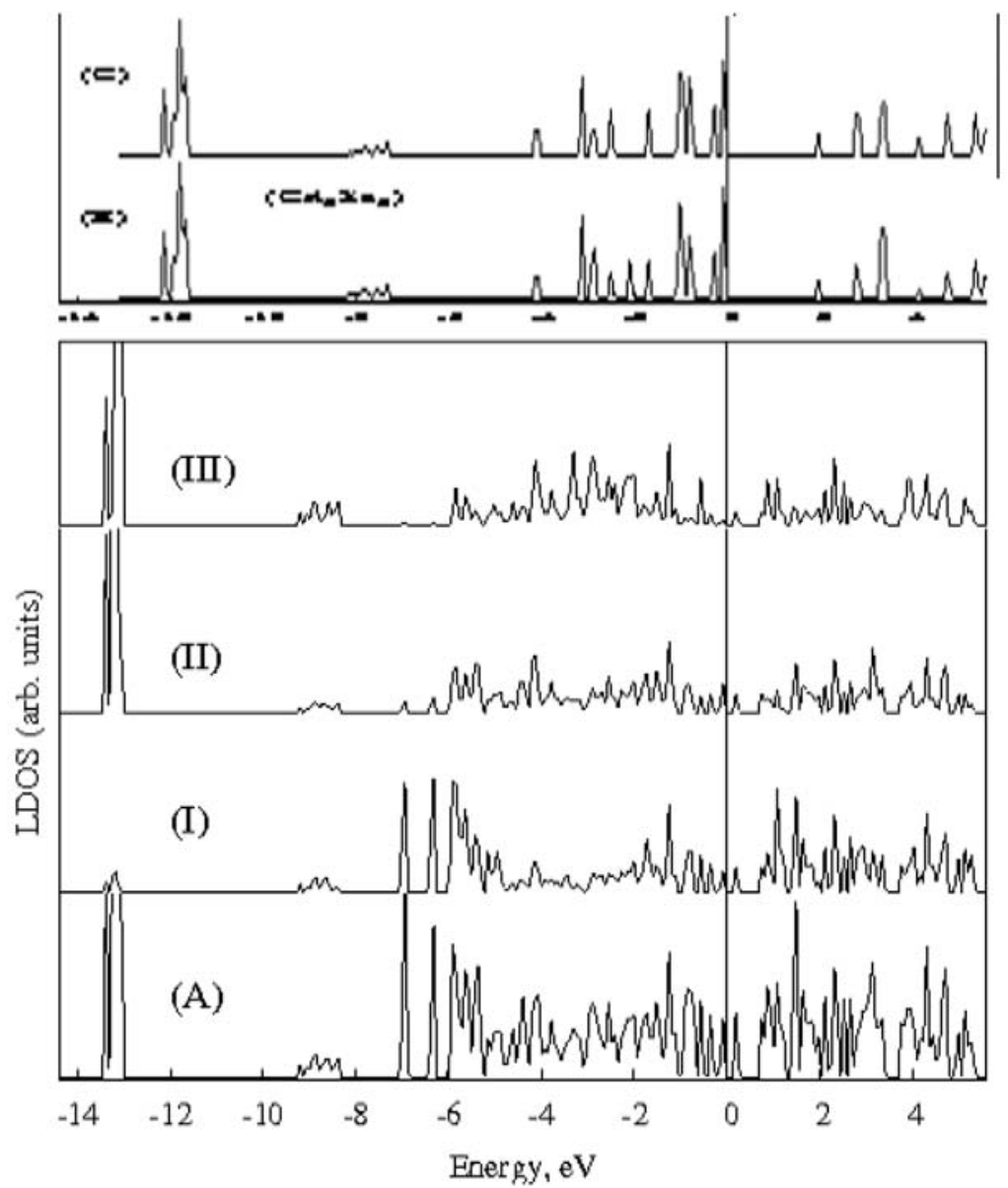

(b)

Figure 2. (a) Geometry of $\mathrm{Au}_{13} \mathrm{Cd}_{6} \mathrm{Se}_{6}$ lead-nanowire. (b) (A) LDOS for $\mathrm{Au}_{13} \mathrm{Cd}_{6} \mathrm{Se}_{6}$ leadnanowire;

\section{(b) $\mathrm{Au}_{13} \mathrm{Cd}_{10} \mathrm{Se}_{10}$ Nanocontact}

Figure 3 (a) depicts the geometry of $\mathrm{Au}_{13} \mathrm{Cd}_{10} \mathrm{Se}_{10}$ nanodumbell. The outward relaxation of $\mathrm{Cd}$ and Se atoms at the nanojunction is now reduced by about $0.13 \AA$, as compared to the value in $\mathrm{Au}_{13} \mathrm{Cd}_{6} \mathrm{Se}_{6}$ nanodumbell. A similar elongation of $\mathrm{Au}-\mathrm{Au}$ bond lengths for those $\mathrm{Au}$ atoms bonded to sulfur are found by Larsson (Larsson et al., 2002). Except at the nanojunction the structure of $\mathrm{Cd}_{10} \mathrm{Se}_{10}$ nanowire is a fragment of the wurtzite bulk structure with an average Au$\mathrm{Cd}$ and Au-Se bond lengths are $2.69 \AA$ and $2.53 \AA$, respectively and average Cd-Se bond length is found to be $2.55 \AA$. While the Se atom is singly bonded, the $\mathrm{Cd}$ atom is found to be doubly bonded to Au atoms. 


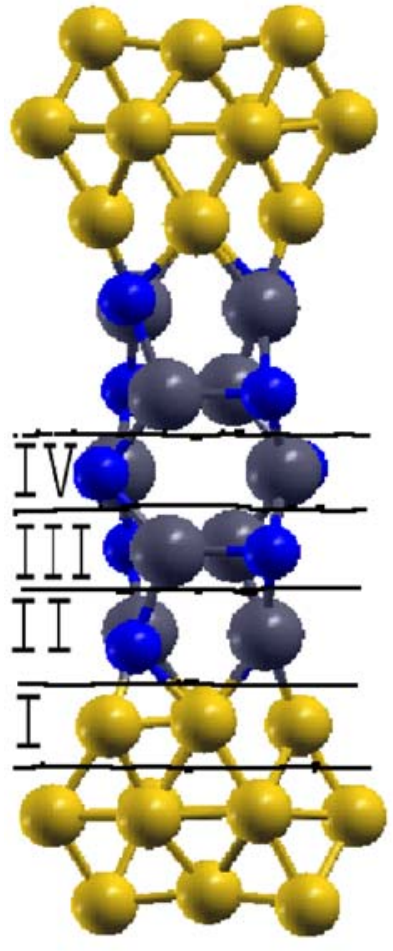

(a)
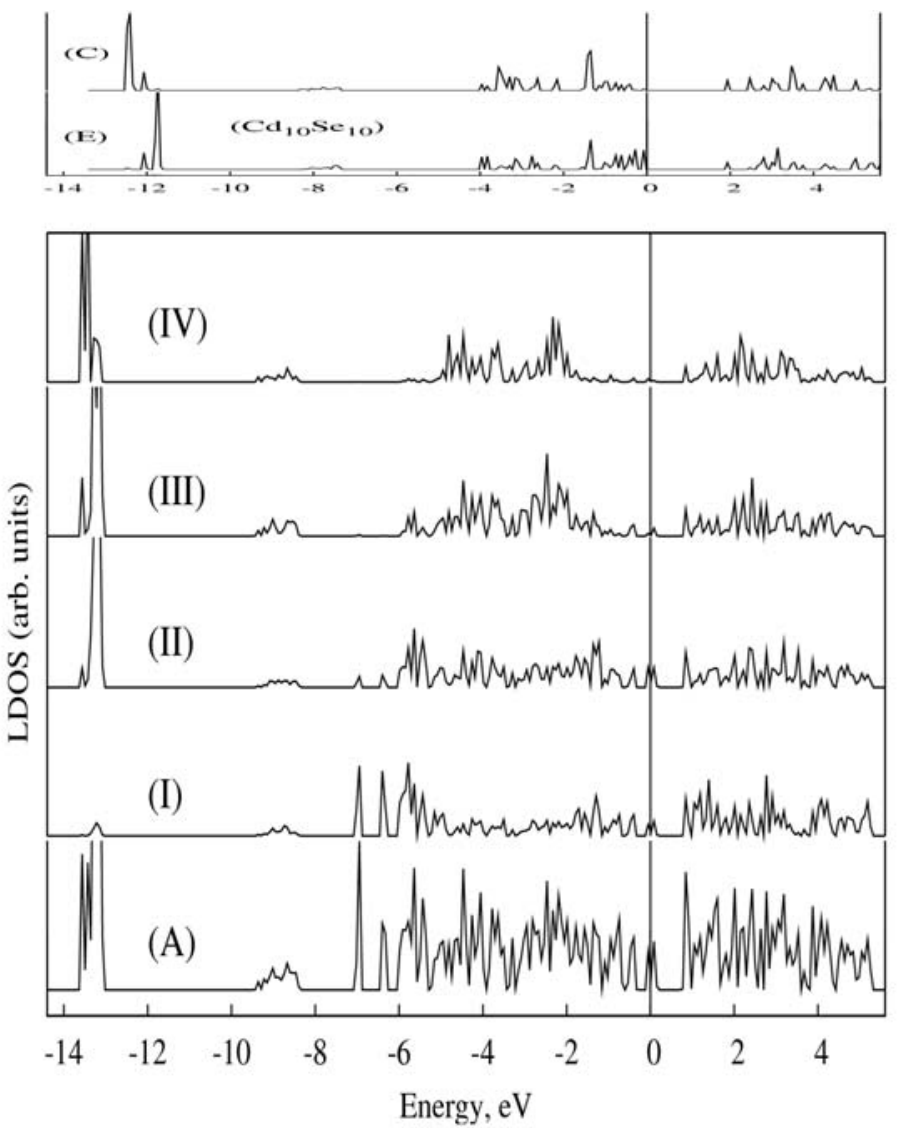

(b)

Figure 3. (a) Geometry of $\mathrm{Au}_{13} \mathrm{Cd}_{10} \mathrm{Se}_{10}$ lead-nanowire. (b): (A) LDOS for $\mathrm{Au}_{13} \mathrm{Cd}_{10} \mathrm{Se}_{10}$ leadnanowire;

(I) - (IV) are LDOS calculated in different regions as defined in the geometry in (a). On top of the LDOS of $\mathrm{Au}_{13} \mathrm{Cd}_{10} \mathrm{Se}_{10}$ NDB is shown the LDOS at the edge (E) and center (C) of bare $\mathrm{Cd}_{10} \mathrm{Se}_{10}$ nanowire plotted for comparison. The discrete spectra were broadened by a Gaussian of width $0.02 \mathrm{eV}$. The vertical line denotes $\mathrm{E}_{\mathrm{F}}$.

Figure 3 (b) displays LDOS calculated in the different regions as defined in the geometry, Fig. 3 (a), where (A) belongs to the LDOS of whole $\mathrm{Au}_{13} \mathrm{Cd}_{10} \mathrm{Se}_{10}$ nanodumbell. As was the case for the $\mathrm{Au}_{13} \mathrm{Cd}_{6} \mathrm{Se}_{6}$ nanodumbell, regions (II) and (III) are fully metalized by the MIGS, while there are no states in region (IV) around the Fermi level. That is, as one moves from the junction, (I), to the center, (IV), of the nanowire along the axis, one observes a widening of the gap around the Fermi level. This is an indication that near the junction CdSe behaves like a metal and as one goes to the center along the axis, it behaves like a semiconductor. The length of the $\mathrm{Cd}_{10} \mathrm{Se}_{10}$ nanowire is $8.76 \AA$. Thus, MIGS almost disappear at about $4.36 \AA$. This distance is less than the 
length of $\mathrm{Cd}_{6} \mathrm{Se}_{6}$ nanowire in $\mathrm{Au}_{13} \mathrm{Cd}_{6} \mathrm{Se}_{6}$ nanodumbell.

The binding energy calculated for the nanodumbell is $7.90 \mathrm{eV}$. From the position of $\mathrm{E}_{\mathrm{F}}$ in the electrode attached wire and the position $\left(\mathrm{E}_{\mathrm{c}}\right)$ of the bottom of the conduction states in the middle section of the wire, we calculate the Schottky barrier (SB) height (Landman et al., 2000) as 0.87 $\pm 0.01 \mathrm{eV}$. This is the barrier height for tunneling through the LUMO for a molecule sandwiched between two metal electrodes. The SB height at the bulk contact between gold and CdSe SC is $0.49 \mathrm{eV}$ (Kirczenow, 2001).

\section{(c) $\mathrm{Au}_{13} \mathrm{Cd}_{14} \mathrm{Se}_{14}$ Nanocontact}

Figure 4 (a) displays the geometry of $\mathrm{Au}_{13} \mathrm{Cd}_{14} \mathrm{Se}_{14}$ nanodumbell. There is no structural change for this nanodumbell as compared to the structure of the $\mathrm{Au}_{13} \mathrm{Cd}_{10} \mathrm{Se}_{10}$ nanodumbell including bond lengths. The length of the $\mathrm{Cd}_{14} \mathrm{Se}_{14}$ nanowire is $12.90 \AA$.

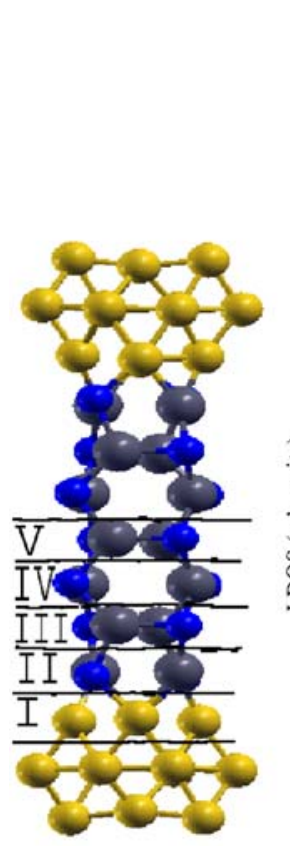

(a)

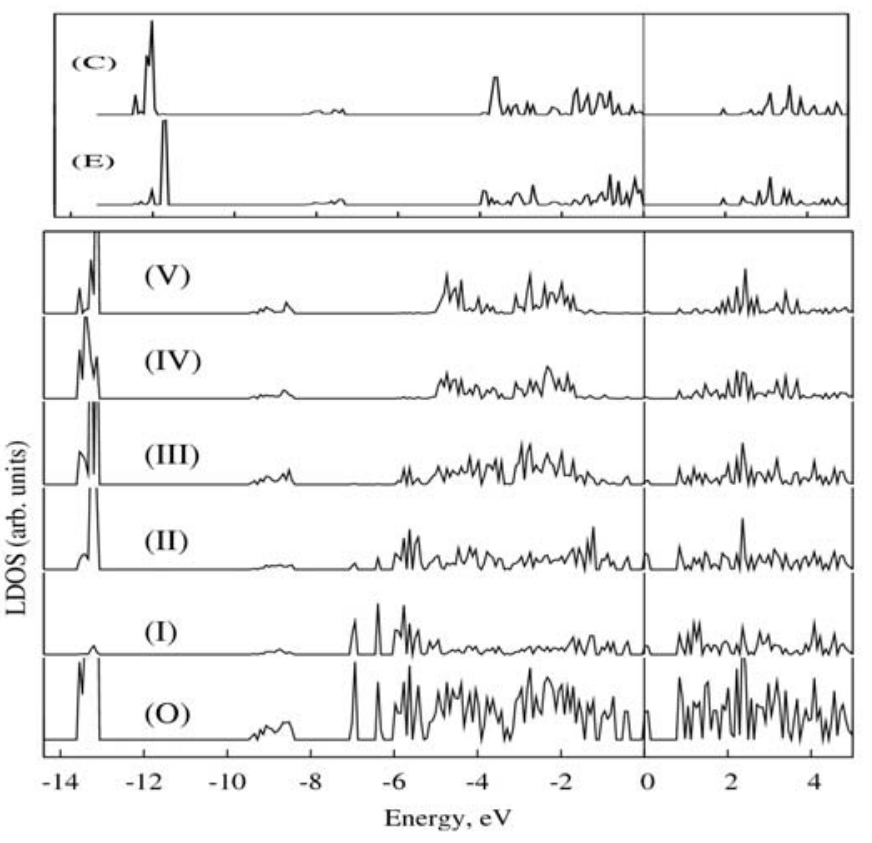

(b)

Figure. 4 (a) Geometry of $\mathrm{Au}_{13} \mathrm{Cd}_{14} \mathrm{Se}_{14}$ lead-nanowir. (b) (A) LDOS for $\mathrm{Au}_{13} \mathrm{Cd}_{14} \mathrm{Se}_{14}$ leadnanowire; (I) - (V) are LDOS calculated in different regions as defined in the geometry (a). On top of the LDOS of $\mathrm{Au}_{13} \mathrm{Cd}_{14} \mathrm{Se}_{14} \mathrm{NDB}$ is shown the LDOS at the edge (E) and center (C) of bare $\mathrm{Cd}_{14} \mathrm{Se}_{14}$ nanowire plotted for comparison. The discrete spectra were broadened by a Gaussian of $0.02 \mathrm{eV}$ width. The vertical line denotes $\mathrm{E}_{\mathrm{F}}$.

The LDOS of $\mathrm{Au}_{13} \mathrm{Cd}_{14} \mathrm{Se}_{14}$ nanodumbell is shown in Fig. 4 (b) where (A) represents the LDOS 
of the whole system. (I) - (V) are LDOS plots calculated in different regions as defined in the geometry. Similar to the case of $\mathrm{Au}_{13} \mathrm{Cd}_{10} \mathrm{Se}_{10}$ nanodumbell, the first two regions of the nanowire, namely regions (II) and (III) are fully metalized as there are shown MIGS in the gap region. However, as we go farther towards the center of the nanowire, the MGS disappear before reaching the center. This happens at a distance of about $4.30 \AA$ from the $\mathrm{Cd} / \mathrm{Se}$ atoms bonded to the Au electrodes. Thus, the disappearance of MGS is closer to the edge than in the case of $\mathrm{Au}_{13} \mathrm{Cd}_{10} \mathrm{Se}_{10}$ nanodumbell. The broader spectrum of LDOS near the nanojunction shrinks as a function of distance from the edge towards the center of the nanowire. This is expected since the participation of the electrons from the Au electrode decreases as one goes towards the center and in this energy range, it is the gold derived states that are contributing to the LDOS.

Binding energy calculated for the nanodumbell is $7.76 \mathrm{eV}$. For the same nanodumbell we have calculated the SB height to be $0.82 \pm 0.01 \mathrm{eV}$. This behavior is confirmed experimentally by Steiner (Steiner et al., 2005) using STM for CdSe and theoretically by Landman (Landman et al., 2000) from $a b$ initio simulations for Si nanorods. The theoretical results show that short (about $6.00 \AA$ ) wires are fully metalized by MIGS resulting in finite conductance. For longer wires, they found a wider gap occurring over a range of about $5 \AA$. Furthermore, they found a gap between the position of $E_{F}$ in the electrode-attached wires and $E_{c}$ of the bottom of the conduction band in the middle of the wire larger by $40 \%$ to $90 \%$ than the SB height at the bulk contact between silicon and aluminum. A qualitative agreement with the theoretical predictions was found experimentally where sub-gap states exist even at much longer distance from the interface. The reason for the longer distance is explained by the researchers (Steiner et al., 2005) as due to the limitation of the spatial resolution of their experiment. This results in unclear topographic image so that the exact location of the interface cannot be predicted.

In Figure 5, panels (i), show the isosurfaces of the electronic charge densities originating from states in the vicinity of the Fermi level calculated for (a) $\mathrm{Au}_{13} \mathrm{Cd}_{6} \mathrm{Se}_{6}$, (b) $\mathrm{Au}_{13} \mathrm{Cd}_{10} \mathrm{Se}_{10}$ and (c) $\mathrm{Au}_{13} \mathrm{Cd}_{14} \mathrm{Se}_{14}$ nanodumbells. Analysis of the charge density, gap, corresponding to states with energies in the gap region (Landman et al., 2000) shows that these MIGS extend into the metal but decay rapidly into the CdSe nanowire. For the short wire gap extends across the entire length, but for the longer wires gap at the interfacial regions closest to the metal electrode differ from those in the middle section of the wires. This can be explained by the occurrence of charge transfer from the metal into the SC (Landman et al., 2000) highly localized at the region of 
contact between them.

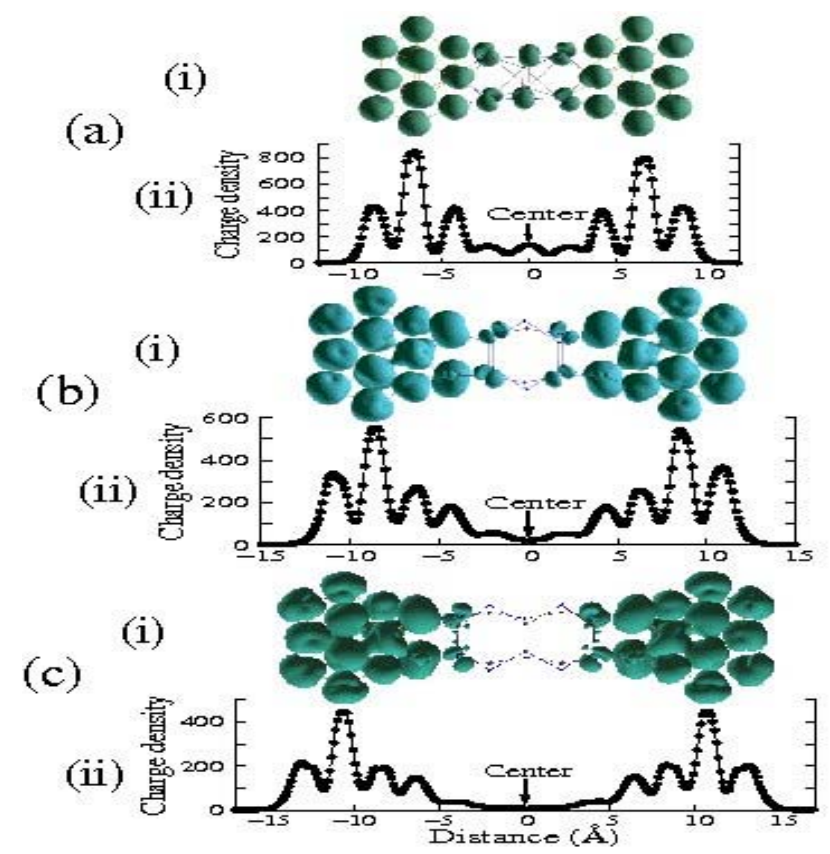

Figure 5. Plots (i) are isosurfaces of the charge densities, gap, calculated for the states in the vicinity of the Fermi level for (a) $\mathrm{Au}_{13} \mathrm{Cd}_{6} \mathrm{Se}_{6}$, (b) $\mathrm{Au}_{13} \mathrm{Cd}_{10} \mathrm{Se}_{10}$ and (c) $\mathrm{Au}_{13} \mathrm{Cd}_{14} \mathrm{Se}_{14}$ nanodumbells respectively\}. Plots (ii) represent the corresponding charge density integrated over a perpendicular surface and shown as a function of distance along the axis of wire.

Figure 5 also displays, in panels (ii), the charge density for the states in the vicinity of the Fermi level, integrated over a surface perpendicular to the axis of nanodumbell and plotted as a function of distance along the axis of the nanodumbell. These plots depict the same structures as those of the corresponding isosurface plots, as expected.

In case of interconnection applications (Belosludov et al., 2004), it is important that the molecular wire should have metallic characteristics. Transport through the longer wires, therefore, requires doping as explained by Landman et al. (2000).

Doping with the metal atoms can modify the electronic structure of the wires enhancing the LDOS in the regions of the wire bonded directly to the metal electrode and the one in the middle of the cluster bridge. Accordingly, we doped $\mathrm{Au}_{13} \mathrm{Cd}_{10} \mathrm{Se}_{10}$ nanodumbell by one interstitial $\mathrm{Au}$ atom at the middle, and $\mathrm{Au}_{13} \mathrm{Cd}_{14} \mathrm{Se}_{14}$ nanodumbell by two $\mathrm{Au}$ atoms placed at equal distance from the center of the nanowire. Geometries and integrated charge densities calculated over a surface perpendicular to the axis plotted as a function of distance along the axes of the wires are shown in figure 6. The doping is seen to enhance the charge density around the Fermi levels of 
the nanodumbells. There is a re-enforcement of contributions from the two Au atoms doped in $\mathrm{Au}_{13} \mathrm{Cd}_{14} \mathrm{Se}_{14}$ nanodumbell as compared to the one $\mathrm{Au}$ atom doped in $\mathrm{Au}_{13} \mathrm{Cd}_{10} \mathrm{Se}_{10}$ nanodumbell. However, in $\mathrm{Au}_{13} \mathrm{Cd}_{14} \mathrm{Se}_{14}$ nanodumbell, we do see a region between the $\mathrm{Au}_{13}$ lead and the doped $\mathrm{Au}$ atoms where the charge density is not affected due to doping. These regions, therefore, require extra Au atom doping.

(a)
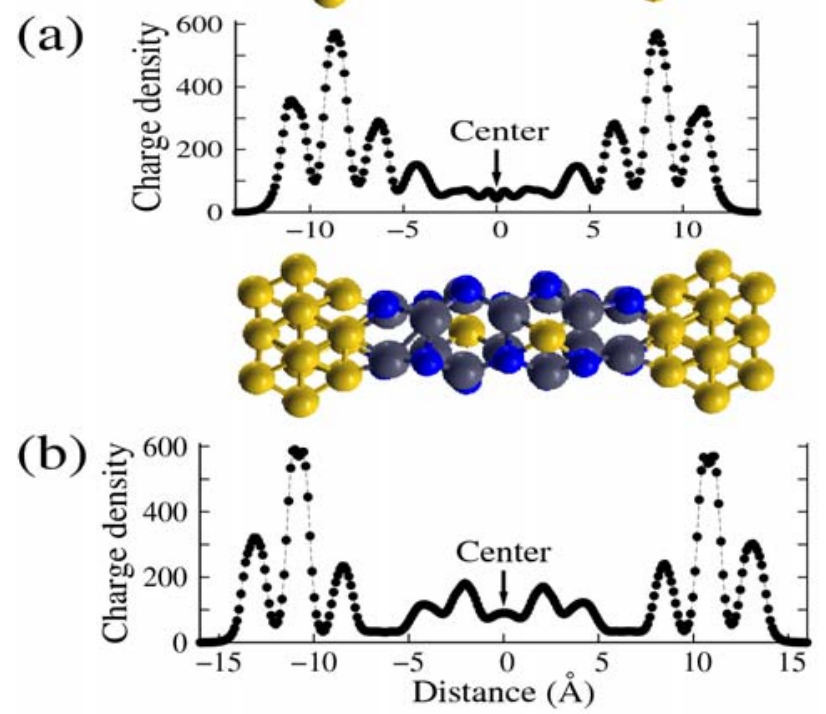

Figure 6. Charge density of the states in the vicinity of the Fermi level integrated over a perpendicular surface as function of distance along the axis of (a) one Au atom doped $\mathrm{Au}_{13} \mathrm{Cd}_{10} \mathrm{Se}_{10}$ and (b) two $\mathrm{Au}$ atom doped $\mathrm{Au}_{13} \mathrm{Cd}_{14} \mathrm{Se}_{14}$ nanowires along with their respective geometries.

\section{CONCLUSION}

CdSe nanowires etched from the bulk wurtzite structures connected to gold electrodes are studied using LDA-DFT. The nanowires considered in the study are $\mathrm{Cd}_{6} \mathrm{Se}_{6}, \mathrm{Cd}_{10} \mathrm{Se}_{10}$ and $\mathrm{Cd}_{14} \mathrm{Se}_{14}$ and $\mathrm{Au}_{13}$ as the electrode. The nanodumbells can provide a unique model system to study the physical properties of metal-SC nanojunctions, a fundamental problem of significant importance in the quest for reliable nanoelectronic devices.

A gap similar to that in bare CdSe nanorods (Katz et al., 2002) is observed near the nanodumbell center, while sub-gap structures emerges near the metal-SC nanocontact. This behavior is attributed to the formation of sub-gap interface states that vanish rapidly towards the center of the wire, consistent with experimental predictions (Steiner et al., 2005). 
For the shortest wire bridging the Au electrodes we find full metalization by MIGS, which results in a finite electronic conductance (Landman et al., 2000), while for longer wires we find Schottky barrier heights larger than the barrier at the corresponding bulk interface by $68 \%$ and $77 \%$ for $\mathrm{Au}_{13} \mathrm{Cd}_{14} \mathrm{Se}_{14}$ and $\mathrm{Au}_{13} \mathrm{Cd}_{10} \mathrm{Se}_{10}$ nanodumbells, respectively.

For the longer wires, the LDOS at the interfacial regions closest to the metal electrode shows a finite DOS in the gap indicating a metallic property with a gradual "emptying" of the gap as a function of distance from the electrode. Inspection of the charge density in the vicinity of the Fermi level also reveals that, for the short wire gap extends across the entire length of the device whereas for the longer wires gap extend into the metal but decays rapidly into the nanowire. Doping with $\mathrm{Au}$ atoms(s) modify the electronic structure of the wires enhancing the LDOS in the regions of the wire. The enhancement of the charge density around the Fermi level, due to doping, is crucial for conductance.

\section{ACKNOWLEDGEMENTS}

I am grateful to The Ministry of Education, Ethiopia, for financing this work. I am also indebted to CDAC, CMS, IUCCA, Pune-India, for the provision of high computing facilities. Thanks are also due to all those friends who helped me during the work.

\section{REFERENCES}

Aviram, A \& Ratner, M.A. 1974. Molecular rectifiers. Chem. Phys. Lett., 29:277.

Belosludov, R.V., Farajian, A.A., Mizuseki, H., Ichinoseki, K and Kawazoe, Y. 2004, Electron Transport in Molecular Enamel Wires. Jpn. J. Appl. Phys., 43:2061.

Ceperley, D.M \& Alder, B.J. 1980. Density Functional Study of Non-Polar Surfaces of Wurtzite CdSe. Phys. Rev. Lett., 45:566.

Csik, I., Russo, S.P \& Mulvaney, P. 2005. Ground State of the Electron Gas by a Stochastic Method. Chem. Phys. Lett., 414:322.

Dabbousi, B.O., Bawendi, M.G., Onitsuka, O \& Rubner, M.F. 1995. Electroluminescence from CdSe quantum dot/polymer composites. Appl. Phys. Lett., 66:1316.

Deglmann, P., Ahlrichs, R \& Tsereteli, K. 2002. Theoretical studies of ligand-free cadmium selenide and related semiconductor clusters. J. Chem. Phys., 116:1585.

Fernàndez, E.M., Soler, J.M., Garzòn, I.L \& Balbàs, L.C. 2004. Trends in the structure and 
bonding of noble metal clusters. Phys. Rev. B, 70:165403.

Furche, F., Ahlrichs, R., Weis, P., Jacob, C., Gilb, S., Bierweiler, T \& Kappes, M.M. 2002. The structures of small gold cluster anions as determined by a combination of ion mobility measurements and density functional calculations. J. Chem. Phys., 117:6982.

Gilb, S., Weis, P., Furche, F., Ahlrichs, R \& Kappes, M.M. 2002. Structures of small gold cluster cations $\left(\mathrm{Aun}^{+}, \mathrm{n}<14\right)$ : Ion mobility measurements versus density functional calculations. J. Chem. Phys., 116: 4094.

Katz, D., Wizansky, T., Millo, O., Rothenberg, E., Mokar, T \& Banin, U. 2002. Size-Dependent Tunneling and Optical Spectroscopy of CdSe Quantum Rods. Phys. Rev. Lett., 89:086801.

Kirczenow, G. 2001. Ideal Spin Filters: A Theoretical Study of Electron Transmission Through Ordered and Disordered Interfaces Between Ferromagnetic Metals and Semiconductors. Phys. Rev. B., 63:054422.

Kress, G \& Furthmüller. 1996. Efficiency of ab-initio total energy calculations for metals and semiconductors using a plane-wave basis set, J., Compt. Mat. Sci., 6, 15; Efficient iterative schemes for ab-initio total energy calculations using a plane-wave basis set. Phys.Rev. B, 54, 11169.

Landman, U., Barnett, R.N., Scherbakov, A.G \& Avouris, Ph. 2000. Metal Semiconductor Nanocontacts: Silicon Nanowire. Phys. Rev. Lett., 85:1958.

Larsson, J.A., Nolan, M \& Greer, J.C. I. J. 2002. Interactions between Thiol Molecular Linkers and the Au13 Nanoparticle. Phys. Chem. B., 106:5931.

Manna, L., Wang, L.W., Cingolani, R \& Alivisato, A.P. 2005. First-Principles Modeling of Unpassivated and Surfactant-Passivated Bulk Facets of Wurtzite CdSe: A Model System for Studying the Anisotropic Growth of CdSe Nanocrystals. J. Phys. Chem. B., 109:6183.

Millo, O., Katz, D., Cao, Y.W \& Banin, U. 2001. Imaging and Spectroscopy of Artificial-Atom States in Core/Shell Nanocrystal Quantum Dots. Phys. Rev. Lett., 86: 5751.

Nir, I.R., Wagner, H.D., Rubinstein, I \& Hodes, G. 2003. Structural Effects in the Electrodeposition of CdSe Quantum Dots on Mechanically Strained Gold. Adv. Funct. Mater.,13:159.

Nitzan, A. 2001. Electron Transmission Through Molecules and Molecular Interfaces. Annu. Rev. 
Phys. Chem., 52:681.

Piccinin, S., Selloni, A., Scandolo, S., Car, R \& Scoles, G. 2003. Electronic properties of metalmolecule-metal systems at zero bias: A periodic density functional study. J. Chem. Phys., 119:6729.

Puzder, A., Williamson, A.J., Gygi, E \& Galli, G. 2004. Self-Healing of CdSe Nanocrystals: First-Principles Calculations. Phys. Rev. Lett., 92:217401.

Reed, M.A., Zhou, C., Muller, C.J., Burgin, T.P \& Tour, J.M. 1997. Conductance of a Molecular Junction. Science, 278:252.

Settler, K. 2002. Handbook of Thin Films Materials- Nanomaterials and magnetic Thin Films. Academic Press, 5, pp. 61.

Shafai, G.S., Shetty, S., Krishnamurty, S., Shah, V \& Kanhere, D.G. 2007. Density functional investigation of the interaction of acetone with small gold clusters. J. Chem. Phys., 126: 014704.

Steiner, D., Mokari, T., Banin, U \& Millo, O. 2005. Electronic Structure of MetalSemiconductor Nanojunctions in Gold CdSe Nanodumbbells. Phys. Rev. Lett., 95:056805.

Stipe, B.C., Rezaei, M.A., Ho, W., Gao, S., Persson, M \& Lundqvist, B.I. 1997. Single-Molecule Dissociation by Tunneling Electrons. Phys. Rev. Lett., 78: 4410.

Thomas, N.L., Herz, E., Schöps, O., Woggon, U \& Artemyev, M.V. 2005. Exciton Fine Structure in Single CdSe Nanorods. Phys. Rev. Lett., 94:016803.

Troparevsky, M.C \& Chelikowsky, J.R. 2001. Structural and Electronic Properties of CdS and CdSe Clusters. J. Chem. Phys., 114:943.

Vanderbilt, D. 1990. Soft Self-consistent Pseudopotentials in a Generalized Eigenvalue Formalism. Phys. Rev. B., 41:7892.

Vosko, S.H., Wilk, L \& Nusair, M. 1980. Accurate spin-dependent electron liquid correlation energies for local spin density calculations: a critical analysis. Can. J. Phys., 58:1200.

Wang, J., Wang, G \& Zhao, J. 2002. Density-functional study of Aun(n=2-20) clusters: Lowestenergy structures and electronic properties. Phys. Rev. B., 66:035418.

Wu, S., Liu, H., Liu, H., Wu, Z., Du, Z \& Schelly, Z.A. 2007. Synthesis and Bandgap Variation of Molecular-Size CdSe Clusters via Electroporation of Vesicles. Nanotechnology, 18(48): 485607(1-6). 
Xia, J.-B., Chang, K \& Li, S.S. 2004. Electronic Structure and optical property of semiconductor nanocrystallites. Compt. Mater. Sci., 30:274.

Yu, M., Fernando, G.W., Li, R., Papadimitrakopoulos, F., Shi, N \& Ramprasad, R. 2006. First principles study of CdSe quantum dots: stability, surface unsaturations, and experimental validation. Appl. Phys. Lett., 88:231910.

Zhou, C., Deshpande, M.R, Reed, M.A, Il, L.J \& Tour, J.H. 1997. Nanoscale Metal/ SelfAssembled Monolayer/Metal Heterostructures. Appl. Phys. Lett.,71:611. 